\title{
On Inverse Generalized Synchronization of Continuous Chaotic Dynamical Systems
}

\author{
Adel Ouannas ${ }^{1}$. Zaid Odibat ${ }^{2}$
}

Published online: 17 March 2015

(C) Springer India Pvt. Ltd. 2015

\begin{abstract}
In this paper, the inverse generalized synchronization problem for different dimensional chaotic dynamical systems in continuous-time is proposed and investigated. New results are derived using new control method and stability theory. Numerical simulations are used to verify the effectiveness of the proposed schemes.
\end{abstract}

Keywords Generalized synchronization · Chaos - Dynamical system · Inverse generalized synchronization

\section{Introduction}

Since the synchronization of chaotic systems is discovered [1,2], the synchronization problem in chaotic systems has been intensively and extensively studied in recent decades [3-7]. Up to now, various methods have been developed to design controllers in the continuous-time dynamical systems such as PC method [5], OGY method [4], active control approach [8,9], adaptive control method [9-11], backstepping design control method [12] and sliding mode control method [13,14], etc.

Complete synchronization [11,15], phase synchronization [17], lag synchronization [18], anticipated synchronization [19] of chaotic systems have been described theoretically and observed experimentally. Complete synchronization which is defined as the coincidence of states of interacting systems; phase synchronization which means entrainment of phases of chaotic oscillators, whereas their amplitudes remain uncorrelated; lag synchronization which

Zaid Odibat

z.odibat@gmail.com; odibat@bau.edu.jo

Adel Ouannas

ouannas_adel@yahoo.fr

1 LAMIS Laboratory, Department of Mathematics and Computer Science, University of Tebessa, Tébessa, Algeria

2 Department of Mathematics, Faculty of Science, Al-Balqa'Applied University, Salt 19117, Jordan 
appears as a coincidence of time-shifted states of two systems; and anticipated synchronization also appears as a coincidence of shifted-in-time states of two coupled systems, but in this case, in contrast to lag synchronization, the driven system anticipates the driver. Another interesting dynamic behavior is the so-called generalized synchronization (GS), where two chaotic systems are said to be synchronized if a functional relationship exists between the states of the two systems [21]. Noting that most presented methods to achieve GS are related to continuous-time systems [15,20-22] and most of them are proposed to investigate the existence of arbitrary boundary function which contains the slave system.

The main aim of the present paper is to describe the inverse problem of generalized synchronization for coupled chaotic systems. We, based on new design method, present a constructive scheme to study inverse generalized synchronization (IGS) between $n$-D and $m$ D continuous chaotic dynamical systems. In order to verify the effectiveness of the proposed method, we apply it to chaotic and hyperchaotic continuous-time systems.

The rest of this paper is arranged as follows: in "Inverse Generalized Synchronization" section, the problem of IGS in continuous-time dynamical systems is investigated. In "Numerical Examples" section, numerical simulations are used to validate theoretical synchronization results. Finally, conclusion remarks are given in "Conclusion" section.

\section{Inverse Generalized Synchronization}

Consider the chaotic system described by

$$
\dot{X}(t)=A X(t)+f(X(t)),
$$

where $X(t)=\left(x_{i}(t)\right)_{1 \leq i \leq n} \in \mathbb{R}^{n}$ is the state vector, $A=\left(a_{i j}\right)$ is the $n \times n$ matrix of parameter system and $f=\left(f_{i}(X(t))\right)_{1 \leq i \leq n} \in \mathbb{R}^{n}$ is the nonlinear part of system (1), as the master system. Then consider the controlled system described by

$$
\dot{Y}(t)=G(Y(t))+U,
$$

where $Y(t)=\left(y_{i}(t)\right)_{1 \leq i \leq m} \in \mathbb{R}^{m}$ is the state vector, $G=\left(G_{i}(Y(t))\right)_{1 \leq i \leq m} \in \mathbb{R}^{m}$ is a continuous vector function and $U=\left(u_{i}\right)_{1 \leq i \leq m} \in \mathbb{R}^{m}$ is the vector controller to be determined, where the system $\dot{Y}(t)=G(Y(t))$ is assumed to be chaotic, as the slave system.

First of all, we call the definition of generalized synchronization then we present the definition of inverse generalized synchronization for coupled chaotic systems given in Eqs. (1) and (2).

Definition 1 The master system (1) and the slave system (2) are said to be generalized synchronized with respect to the vector map $\phi$ if there exists a controller $U=\left(u_{i}\right)_{1 \leq i \leq m} \in$ $\mathbb{R}^{m}$ and a given map $\phi: \mathbb{R}^{n} \longrightarrow \mathbb{R}^{m}$ such that the synchronization error

$$
e(t)=Y(t)-\phi(X(t)),
$$

satisfies that $\lim _{t \rightarrow+\infty}\|e(t)\|=0$.

Remark 1 Generalized synchronization of chaotic dynamical systems with different dimensions, based on Lyapunov stability theory, have been studied and carried out, for example, in Refs. [20,21].

Definition 2 The master system (1) and the slave system (2) are said to be inverse generalized synchronized with respect to the vector map $\phi$ if there exists a controller $U=\left(u_{i}\right)_{1 \leq i \leq m} \in$ $\mathbb{R}^{m}$ and a given map $\phi: \mathbb{R}^{m} \longrightarrow \mathbb{R}^{n}$ such that the synchronization error 


$$
e(t)=X(t)-\phi(Y(t)),
$$

satisfies that $\lim _{t \rightarrow+\infty}\|e(t)\|=0$.

In order to study the IGS of continuous dynamical systems given in Eqs. (1) and (2), we discuss the asymptotical stable of zero solution of synchronization error system $e(t)=$ $X(t)-\phi(Y(t))$. We design the controller $U$ such that the solutions of the error system $e_{i}(t)$ go to $0, i=1,2, \ldots, n$, as $t$ goes to $+\infty$. The error system can be derived as

$$
\dot{e}(t)=(A-K) e(t)+R-D \phi(Y(t)) U,
$$

where $D \phi(Y(t)) \in \mathbb{R}^{n \times m}$ is the Jacobian matrix of the map $\phi(Y(t))$,

$$
\begin{gathered}
D \phi(Y(t))=\left(\begin{array}{cccc}
\frac{\partial \phi_{1}}{\partial y_{1}} & \frac{\partial \phi_{1}}{\partial y_{2}} & \cdots & \frac{\partial \phi_{1}}{\partial y_{m}} \\
\frac{\partial \phi_{2}}{\partial y_{1}} & \frac{\partial \phi_{2}}{\partial y_{2}} & \cdots & \frac{\partial \phi_{2}}{\partial y_{m}} \\
\vdots & \vdots & \ddots & \vdots \\
\frac{\partial \dot{\phi}_{n}}{\partial y_{1}} & \frac{\partial \dot{\phi}_{n}}{\partial y_{2}} & \cdots & \frac{\partial \dot{\phi}_{n}}{\partial y_{m}}
\end{array}\right), \\
R=K X(t)+(A-K) \phi(Y(t))+f(X(t))-D \phi(Y(t)) g(Y(t)),
\end{gathered}
$$

and $K=\left(k_{i j}\right)_{n \times n}$ is unknown control matrix to be determined.

\section{Case I: $n<m$}

In this case, to achieve synchronization between the master system (1) and the slave system (2), we choose the controller $U$ as

$$
U=\left(u_{1}, \ldots, u_{n}, 0, \ldots, 0\right)^{T} \in \mathbb{R}^{m},
$$

then the error systems (5) can be written as

$$
\dot{e}(t)=(A-K) e(t)+R-J_{1} U_{1},
$$

where

$$
J_{1}=\left(\begin{array}{cccc}
\frac{\partial \phi_{1}}{\partial y_{1}} & \frac{\partial \phi_{1}}{\partial y_{2}} & \cdots & \frac{\partial \phi_{1}}{\partial y_{n}} \\
\frac{\partial \phi_{2}}{\partial y_{1}} & \frac{\partial \phi_{2}}{\partial y_{2}} & \cdots & \frac{\partial \phi_{2}}{\partial y_{n}} \\
\vdots & \vdots & \ddots & \vdots \\
\frac{\partial \phi_{n}}{\partial y_{1}} & \frac{\partial \phi_{n}}{\partial y_{2}} & \cdots & \frac{\partial \phi_{n}}{\partial y_{n}}
\end{array}\right),
$$

and $U_{1}=\left(u_{i}\right)_{1 \leq i \leq n}$.

Theorem 1 For an invertible matrix $J_{1}$, the master system (1) and the slave system (2) are globally inverse generalized synchronized with respect to $\phi$ if the following conditions are satisfied

(i) $U_{1}=J_{1}^{-1} R$.

(ii) $(K-A)^{T}+(K-A)$ is a positive definite matrix.

Proof 1 By substituting the control law (i) into Eq.(9), the error system can be written as

$$
\dot{e}(t)=(A-K) e(t) .
$$


Construct the candidate Lyapunov function in the form

$$
V(e(t))=e^{T}(t) e(t),
$$

we obtain

$$
\begin{aligned}
\dot{V}(e(t)) & =\dot{e}^{T}(t) e(t)+e^{T}(t) \dot{e}(t) \\
& =e^{T}(t)(A-K)^{T} e(t)+e^{T}(t)(A-K) e(t) \\
& =e^{T}(t)\left[(A-K)^{T}+(A-K)\right] e(t) .
\end{aligned}
$$

Using (ii), we get $\dot{V}(e(t))<0$. Thus, from the Lyapunov stability theory, it is immediate that all solutions of error system (11) go to zero as $t \rightarrow \infty$. Therefore, systems (1) and (2) are globally inverse generalized synchronized.

\section{Case II: $m<n$}

Now, the error system between the master system (1) and the slave system (2), given in Eq. (5), can be written in scalar form as

$$
\dot{e}_{i}(t)=\sum_{j=1}^{n}\left(a_{i j}-k_{i j}\right) e_{j}(t)+R_{i}-\tilde{u}_{i}, \quad 1 \leq i \leq n,
$$

where

$$
\begin{aligned}
& R_{i}=\sum_{j=1}^{n} k_{i j} x_{j}(t)+\sum_{j=1}^{n}\left(a_{i j}-k_{i j}\right) \phi_{j}(Y(t))+f_{i}(X(t))-\sum_{j=1}^{m} \frac{\partial \phi_{i}}{\partial y_{j}} g_{j}(Y(t)), \quad 1 \leq i \leq n, \\
& \tilde{u}_{i}=\sum_{j=1}^{m} \frac{\partial \phi_{i}}{\partial y_{j}} u_{j}, \quad 1 \leq i \leq n .
\end{aligned}
$$

In this case, to achieve synchronization between systems (1) and (2), we assume that $\frac{\partial \phi_{i}}{\partial y_{j}}$, $1 \leq j \leq m$, are not all equal zero. Therefore, the error system described in Eq. (13) can be written in compact form as

$$
\dot{e}(t)=(A-K) e(t)+R-U_{2},
$$

where $U_{2}=\left(\tilde{u}_{i}\right)_{1 \leq i \leq n}$.

Theorem 2 The master system (1) and the slave system (2) are globally inverse generalized synchronized with respect to $\phi$ if the following conditions are satisfied

(i) $U_{2}=R$.

(ii) All eigenvalues of $A-K$ have negative real parts.

Proof 2 By substituting the control law (i) into Eq. (16), the error system can be described as

$$
\dot{e}(t)=(A-K) e(t) .
$$

Thus, by asymptotic stability of linear continuous-time systems, if all eigenvalues of $A-K$ have negative real parts, it is immediate that all solutions of error system (17) go to zero as $t \rightarrow \infty$. Therefore, systems (1) and (2) are globally inverse generalized synchronized. 
Fig. 1 Chaotic attractor of Lorenz system when $(\alpha, \gamma, \beta)=(10,28,8 / 3)$

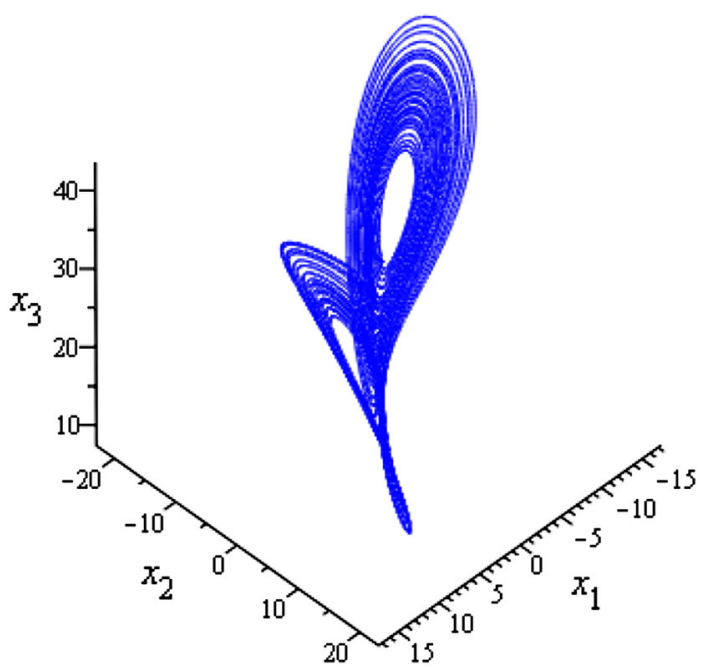

\section{Numerical Examples}

In order to show the effectiveness of the presented approach of synchronization, two numerical examples are used to discuss the issue of realizing IGS between $n$-D and $m$-D continuous chaotic dynamical systems.

\section{Example 1: $(n<m)$}

Here we consider Lorenz system as the master system and the controlled hyperchaotic Liu system as the slave system. Lorenz system can be described as

$$
\left\{\begin{array}{l}
\dot{x}_{1}=\alpha\left(x_{2}-x_{1}\right) \\
\dot{x}_{2}=\gamma x_{1}-x_{2}-x_{1} x_{3} \\
\dot{x}_{3}=-\beta x_{3}+x_{1} x_{2}
\end{array}\right.
$$

which has a chaotic attractor, for example, when $(\alpha, \gamma, \beta)=(10,28,8 / 3)$ [25]. The Lorenz chaotic attractor is shown in Fig. 1. The controlled hyperchaotic Liu system can be described as

$$
\left\{\begin{array}{l}
\dot{y}_{1}=a\left(y_{2}-y_{1}\right)+u_{1} \\
\dot{y}_{2}=b y_{1}+y_{1} y_{3}-y_{4}+u_{2} \\
\dot{y}_{3}=-c y_{3}-y_{1} y_{2}+y_{4}+u_{3} \\
\dot{y}_{4}=d y_{1}+y_{2}+u_{4}
\end{array},\right.
$$

where $U=\left(u_{1}, u_{2}, u_{3}, u_{4}\right)^{T}$ is the vector controller. The hyperchaotic Liu system has a chaotic attractor, for example, when $(a, b, c, d)=(10,35,1.4,5)$ [24]. The projections of hyperchaotic Liu chaotic attractor are shown in Fig. 2.

Taking $(\alpha, \gamma, \beta)=(10,28,8 / 3)$ and $(a, b, c, d)=(10,35,1.4,5)$ then Lorenz system and hyperchaotic Liu system exhibit chaotic attractor. If we select the map $\phi: \mathbb{R}^{4} \longrightarrow \mathbb{R}^{3}$ as

$$
\phi\left(y_{1}, y_{2}, y_{3}, y_{4}\right)=\left(y_{1}+y_{2}+y_{4}, y_{2}+y_{3}+y_{4}, y_{3}+y_{4}\right),
$$



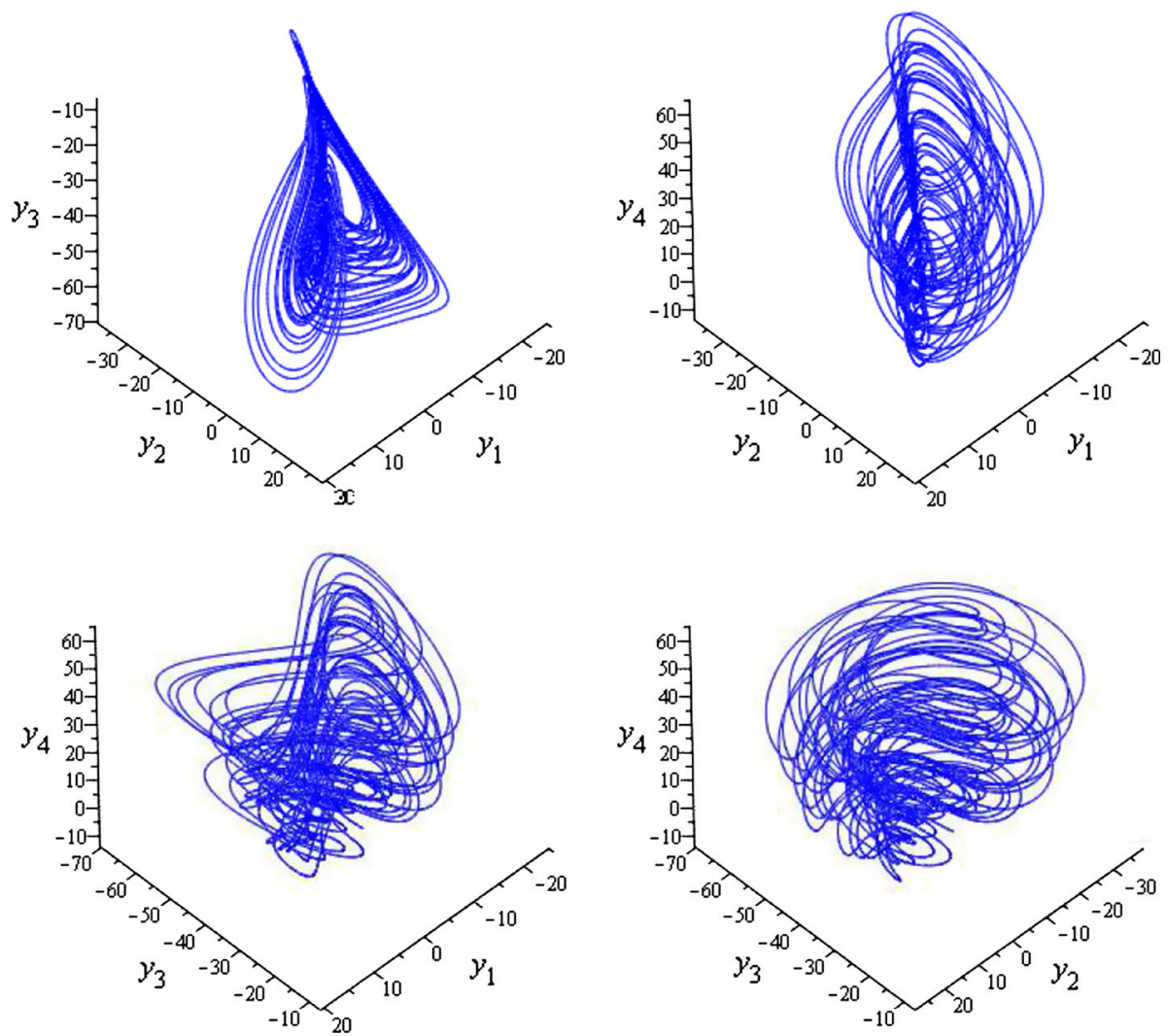

Fig. 2 Chaotic attractor projections of hyperchaotic Liu system when $(a, b, c, d)=(10,35,1.4,5)$

and the control matrix $K$ as

$$
K=\left(\begin{array}{ccc}
0 & 10 & 0 \\
28 & 0 & 0 \\
0 & 0 & 0
\end{array}\right)
$$

Then, according to our approach presented in the previous section, we obtain

$$
\begin{aligned}
D \phi(Y(t)) & =\left(\begin{array}{cccc}
1 & 1 & 0 & 1 \\
0 & 1 & 1 & 1 \\
0 & 0 & 1 & 1
\end{array}\right), \\
A & =\left(\begin{array}{ccc}
-10 & 10 & 0 \\
28 & -1 & 0 \\
0 & 0 & -8 / 3
\end{array}\right),
\end{aligned}
$$

and

$$
f(X(t))=\left(\begin{array}{c}
0 \\
-x_{1} x_{3} \\
x_{1} x_{2}
\end{array}\right) .
$$




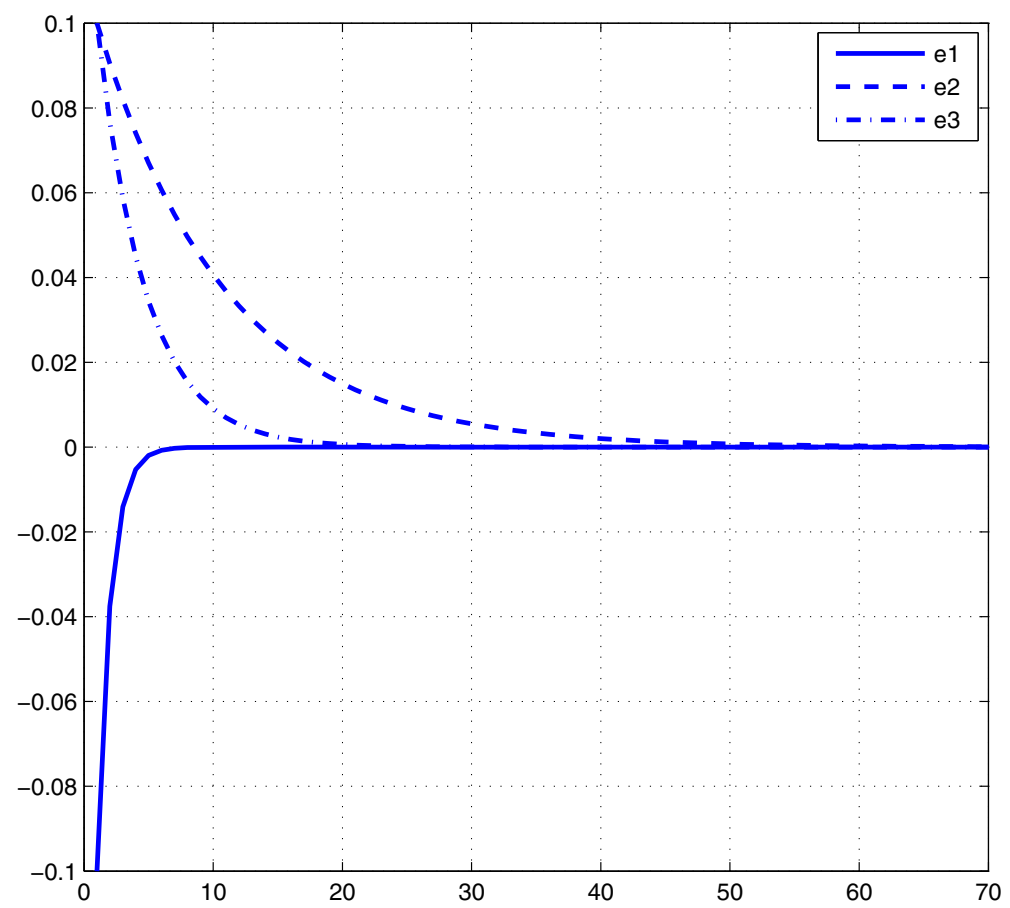

Fig. 3 Time evolution of synchronization errors of systems (18) and (19)

Using simple calculations, we can show that $(K-A)^{T}+(K-A)$ is a positive definite matrix. Therefore, in this case, systems (18) and (19) are inverse generalized synchronized. The error functions evolution is shown in Fig. 3.

\section{Example 2: $(m<n)$}

Now, we consider hyperchaotic Cai system as the master system and the controlled Rössler system as the slave system. Hyperchaotic Cai system can be described as

$$
\left\{\begin{array}{l}
\dot{x}_{1}=a\left(x_{2}-x_{1}\right) \\
\dot{x}_{2}=b x_{1}+c x_{2}-x_{1} x_{3} \\
\dot{x}_{3}=x_{2}^{2}-d x_{4} \\
\dot{x}_{4}=-e x_{1}
\end{array}\right.
$$

which has a chaotic attractor, for example, when $(a, b, c, d, e)=(27.5,3,19.3,2.9,3.3)$ [26]. The projections of Cai system chaotic attractor is shown in Fig. 4. The controlled Rössler system can be described as

$$
\left\{\begin{array}{l}
\dot{y}_{1}=-y_{2}-y_{3}+u_{1} \\
\dot{y}_{2}=y_{1}+\alpha y_{2}+u_{2} \\
\dot{y}_{3}=\gamma+y_{3}\left(y_{1}-\beta\right)+u_{3}
\end{array},\right.
$$

where $U=\left(u_{1}, u_{2}, u_{3}\right)^{T}$ is the vector controller. The Rössler system has a chaotic attractor, for example, when $(\alpha, \gamma, \beta)=(0.2,0.2,5.7)$ [27]. The Rössler chaotic attractor is shown in Fig. 5. 

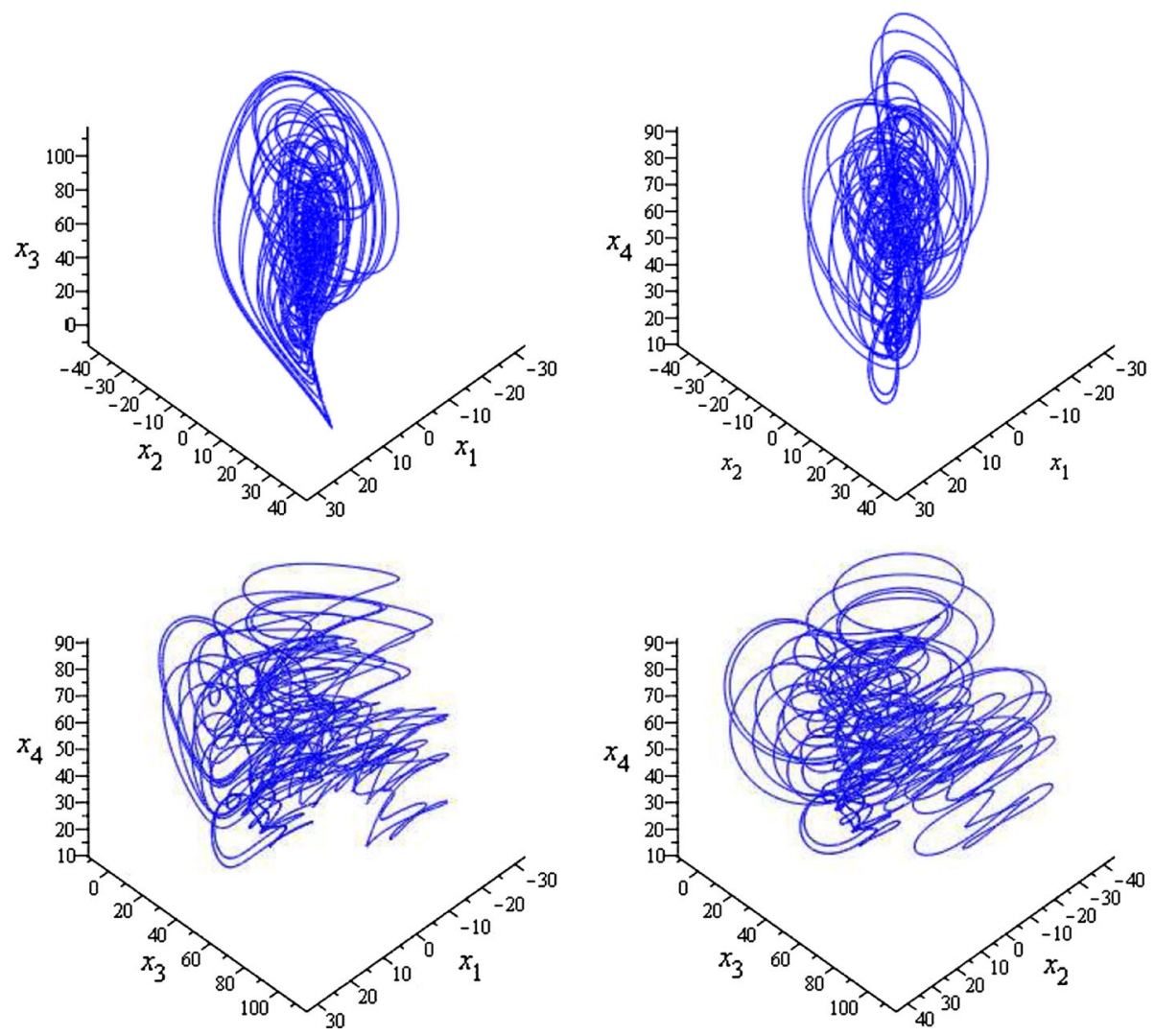

Fig. 4 Chaotic attractor projections of hyperchaotic Cai system when $(a, b, c, d, e)=(27.5,3,19.3,2.9,3.3)$

Fig. 5 Chaotic attractor of Rössler system when $(\alpha, \gamma, \beta)=(0.2,0.2,5.7)$

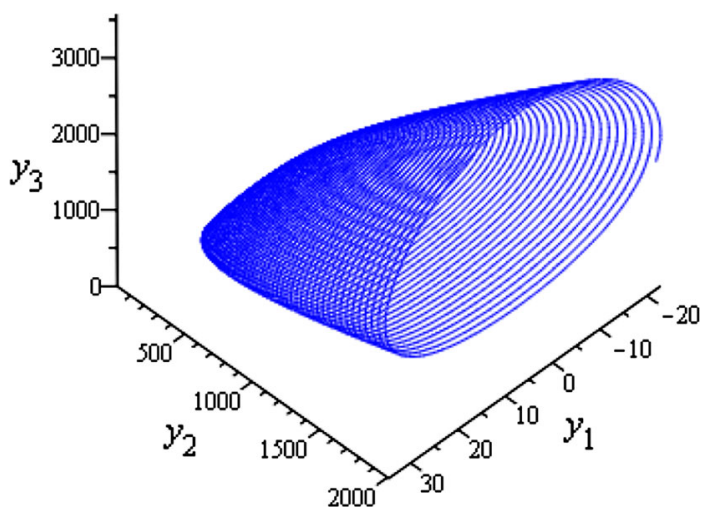

Taking $(a, b, c, d, e)=(27.5,3,19.3,2.9,3.3)$ and $(\alpha, \gamma, \beta)=(0.2,0.2,5.7)$ then hyperchaotic Cai system and Rössler system exhibit chaotic attractor. If we select the map $\phi: \mathbb{R}^{3} \longrightarrow \mathbb{R}^{4}$ as

$$
\phi\left(y_{1}, y_{2}, y_{3}\right)=\left(y_{1}+y_{3}, y_{2}+y_{3}, y_{1}+y_{2}, y_{1}+y_{2}+y_{3}\right),
$$




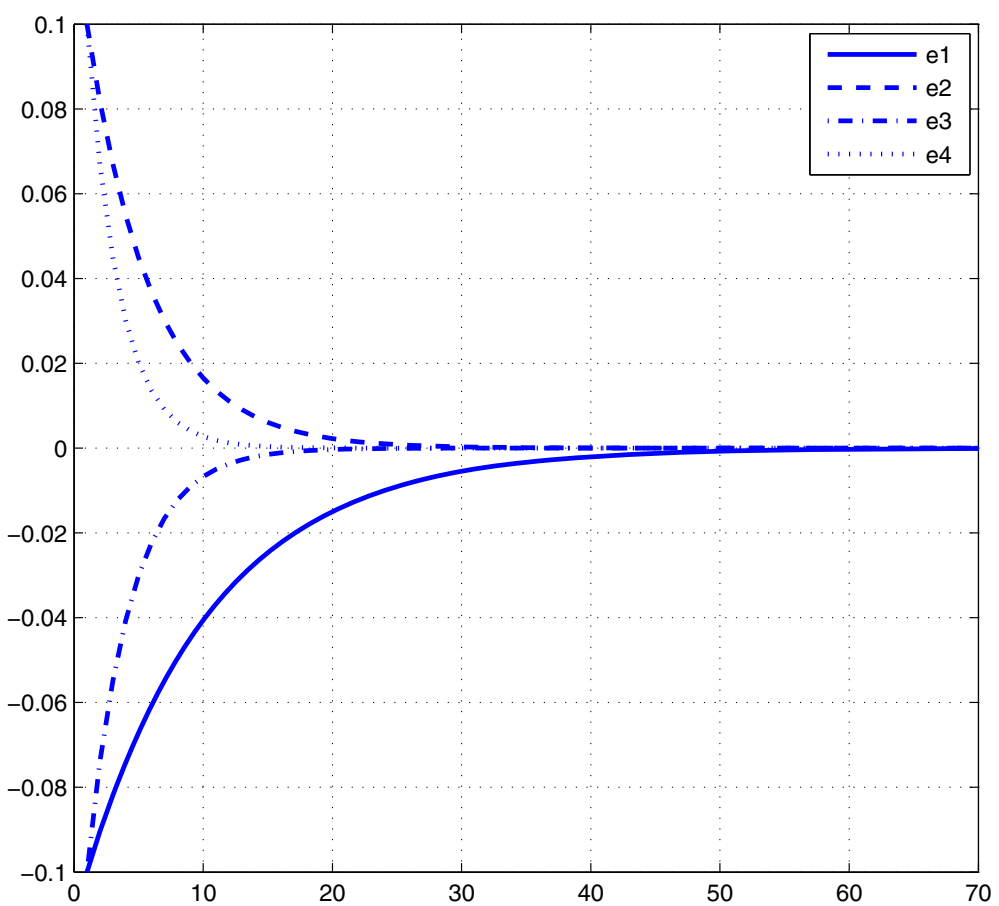

Fig. 6 Time evolution of synchronization errors of systems (25) and (26)

and the control matrix $K$ as

$$
K=\left(\begin{array}{cccc}
-26.5 & 27.5 & 0 & 0 \\
3 & 21.3 & 0 & 0 \\
0 & 0 & 3 & -2.9 \\
-3.3 & 0 & 0 & 4
\end{array}\right)
$$

Then, according to our approach presented in the previous section, we obtain

$$
\begin{aligned}
D \phi(Y(t)) & =\left(\begin{array}{lll}
1 & 0 & 1 \\
0 & 1 & 1 \\
1 & 1 & 0 \\
1 & 1 & 1
\end{array}\right), \\
A & =\left(\begin{array}{cccc}
-27.5 & 27.5 & 0 & 0 \\
3 & 19.3 & 0 & 0 \\
0 & 0 & 0 & -2.9 \\
-3.3 & 0 & 0 & 0
\end{array}\right)
\end{aligned}
$$

and

$$
f(X(t))=\left(\begin{array}{c}
0 \\
-x_{1} x_{3} \\
x_{2}^{2} \\
0
\end{array}\right)
$$


Simply, we can show that all eigenvalues of $A-K$ have negative real parts. Therefore, in this case, systems (25) and (26) are inverse generalized synchronized. The error functions evolution is shown in Fig. 6.

\section{Conclusion}

In this paper, the inverse problem of generalized synchronization of chaotic dynamical systems with different dimensions was investigated. Based on the Lyapunov stability theory and stability theory of linear contious-time dynamical systems, a new method to realize IGS for two different dimensional chaotic systems was proposed. The validity of this approach was verified theoretically and numerically.

\section{References}

1. Yamada, T., Fujisaka, H.: Stability theory of synchronized motion in coupled-oscillator systems. Prog. Theor. Phys. 70, 1240-1248 (1983)

2. Afraimovich, V., Verichev, N., Rabinovich, M.: Stochastic synchronization of oscillations in dissipative systems. Radiophys. Quantum Electron 29, 795-803 (1986)

3. Pecora, L., Carrol, T.: Synchronization in chaotic systems. Phys. Rev. Lett. 64, 821-824 (1990)

4. Ott, E., Grebogi, C., Yorke, J.: Controlling chaos. Phys. Rev. Lett. 64, 1196-1199 (1990)

5. Pyragas, K.: Continuous control of chaos by self-controlling feedback. Phys. Lett. A 170, 421-428 (1992)

6. Boccaletti, S., Kurths, J., Osipov, G., Valladares, D., Zhou, C.: The synchronization of chaotic systems. Phys. Rep. 366, 1-101 (2002)

7. Lu, J., Wu, X., Han, X., Lu, J.: Adaptive feedback synchronization of unified chaotic systems. Phys. Lett. A 329, 327-333 (2004)

8. Feng, C.-F.: Projective synchronization between two different time-delayed chaotic systems using active control approach. Nonlinear Dyn. 62, 453-459 (2010)

9. Zhang, X., Zhu, H.: Anti-synchronization of two different hyperchaotic systems via active and adaptive control. Int. J. Nonlinear Sci. 6, 216-223 (2008)

10. Chen, S., Lu, J.: Synchronization of an uncertain unified systems via adaptive control. Chaos Soliton Fractal 14, 643-647 (2002)

11. Li, X., Leung, A., Han, X., Liu, X., Chu, Y.: Complete (anti-)synchronization of chaotic systems with fully uncertain parameters by adaptive control. Nonlinear Dyn. 63, 263-275 (2011)

12. Zhao, J., Lu, J.: Parameter identification and backstepping control of uncertain Lu system. Chaos Soliton Fractal 17, 721-729 (2003)

13. Yau, H.: Design of adaptive sliding mode controller for chaos synchronization with uncertainties. Chaos Soliton Fractal 22, 341-347 (2004)

14. Yang, C., Lin, C.: Robust adaptive sliding mode control for synchronization of space-clamped FitzHughNagumo neurons. Nonlinear Dyn. 69, 2089-2096 (2012)

15. Banerjee, T., Biswas, D., Sarkar, B.: Complete and generalized synchronization of chaos and hyperchaos in a coupled first-order time-delayed system. Nonlinear Dyn. 71, 279-290 (2013)

16. Cai, G., Hu, P., Li, Y.: Modified function lag projective synchronization of a financial hyperchaotic system. Nonlinear Dyn. 69, 1457-1464 (2012)

17. Feng, C., Lei, X., Chun-Guang, L.: Wavelet phase synchronization of fractional-order chaotic systems. Chin. Phys. Lett. 29, 070501 (2012)

18. Qun, L., Hai-Peng, P., Ling-Yu, X., Xian, Y.: Lag synchronization of coupled multidelay systems. Math. Prob. Eng. 2012, 106830 (2012)

19. Zhong-Kui, S., Wei, X., Xiao-Li, Y.: New scheme of anticipating synchronization for arbitrary anticipation time and its application to long-term prediction of chaotic states. Chin. Phys. B 16(11), 3226-3230 (2007)

20. Wang, Y., Guan, Z.: Generalized synchronization of continuous chaotic systems. Chaos Soliton Fractal 27, 97-101 (2006)

21. Zhang, G., Liu, Z., Ma, Z.: Generalized synchronization of different dimensional chaotic dynamical systems. Chaos Soliton Fractal 32, 773-779 (2007) 
22. Li, X.: Generalized projective synchronization using nonlinear control method. Int. J. Nonlinear Sci. 8, 79-85 (2009)

23. Gao, T., Chen, G., Chen, Z., Cang, S.: The generation and circuit implementation of a new hyperchaos based upon Lorenz system. Phys. Lett. A 361, 78-86 (2007)

24. Liu, L., Liu, C., Zhang, Y.: Analysis of a novel four-dimensional hyperchaotic system. Chin. J. Phys. 46, 386-393 (2008)

25. Lorenz, E.N.: Deterministic nonperiodic flows. J. Atmos. Sci. 20, 130-141 (1963)

26. Wang, H., Cai, G.: Controlling hyperchaos in a novel hyperchaotic system. J. Inf. Comput. Sci. 4, 251-258 (2009)

27. Rössler, O.E.: An equation for continuous chaos. Phys. Lett. A 57, 397-398 (1976) 